Revue d'histoire de l'Amérique française

AG REVUE D.HISTOIRE DE L'AMÉRIQUE FRANÇAISE

\title{
La rébellion de 1837-38 et l'essor du protestantisme canadien-français
}

\section{René Hardy}

Volume 29, numéro 2, septembre 1975

URI : https://id.erudit.org/iderudit/303440ar

DOI : https://doi.org/10.7202/303440ar

Aller au sommaire du numéro

Éditeur(s)

Institut d'histoire de l'Amérique française

ISSN

0035-2357 (imprimé)

1492-1383 (numérique)

Découvrir la revue

Citer cet article

Hardy, R. (1975). La rébellion de 1837-38 et l'essor du protestantisme canadien-français. Revue d'histoire de l'Amérique française, 29(2), 163-189. https://doi.org/10.7202/303440ar d'utilisation que vous pouvez consulter en ligne.

https://apropos.erudit.org/fr/usagers/politique-dutilisation/ 


\title{
LA RÉBELLION DE 1837-38 ET L'ESSOR DU PROTESTANTISME CANADIEN-FRANÇAIS *
}

\author{
ReNÉ HaRdy \\ Département des sciences humaines \\ Université du Québec \\ à Trois-Rivières
}

Au lendemain de la rébellion de 1837-38, le développement rapide des structures de l'Eglise et des effectifs ecclésiastiques fut certes redevable à la personnalité entreprenante de l'évêque Bourget et à l'évolution de la politique britannique permettant alors à l'Eglise d'agir sans craindre l'intervention de l'Etat. Mais au-delà de cette coïncidence de la rencontre d'une conjoncture politique favorable et d'un protagoniste zélé, le dynamisme de l'Eglise bas-canadienne fut aiguillonné par le prosélytisme protestant et par la nécessité de reconquérir auprès des fidèles son influence passablement amoindrie par la propagande laïciste et libérale pré-révolutionnaire.

Le clergé, dirigé en cela par Mgr Bourget, sentit alors l'urgence de mieux encadrer la population et de diversifier ses sphères d'activité. Cette solution lui était assez clairement indiquée par les événements, entre autres par le fait que les protestants disaient ouvertement vouloir profiter de la diminution de l'influence du clergé catholique pour s'implanter. En l'apprenant, Mgr Lartigue, prédécesseur de Bourget, avait écrit à ses curés: “[...] vous faire connaître le mal, c'est assez vous en indiquer le remède, $[\ldots]$ vous veillerez si bien sur votre troupeau pour le préserver de la fureur des loups [...]"1

Le protestantisme présentait alors une double menace: non seulement il mettait en danger la foi des Canadiens, mais il risquait d'ébranler par ses enseignements la position sociale du clergé tout comme l'idéologie libérale l'avait fait dans la décennie 1830. Sous cet aspect, libéralisme et protestantisme étaient confondus. Le clergé, imbu de la pensée des théoriciens de la contrerévolution, de Maistre et de Bonald, avait acquis la conviction que l'un n'était que l'aboutissement de l'autre. "Le XVIe et le

* Mon collègue Thierry Ruddell a participé à cette recherche qui s'inscrit dans un projet plus vaste devant faire l'objet bientôt d'une publication commune.

1 Circulaire du 21 juillet 1839, citée par Léon Pouliot, La réaction catholique de Montréal, 1840-41 (Montréal, 1942), 7.

[163]

RHAF, vol. 29, no 2 (septembre 1975) 
XVIIe siècles, d'affirmer de Maistre, pourraient être nommés les prémisses du XVIIIe, qui ne fut en effet que la conclusion des deux précédents [...]"2. Réforme, philosophisme, révolution, croyait-on, s'enchaînaient sur la base de la valorisation du "jugement privé" entraînant du même coup l'anéantissement d'une conception de l'autorité défendue par l'Eglise et sur laquelle reposait en partie sa fonction sociale. Il leur suffisait de jeter un certain regard sur la réalité canadienne pour se convaincre de la justesse de ce rapprochement: le patriote Côté se convertissait au protestantisme; Jean-Baptiste-Eric Dorion ouvrait ses colonnes de L'Avenir à l'évangéliste suisse Jean Vernier; l'Institut canadien comptait parmi ses membres actifs les baptistes canadiens-français Narcisse Cyr et Théodore Lafleur et, disait-on, encourageait la venue à Montréal de l'apostat Chiniquy ; les journaux conservateurs reprochaient au Pays d'annoncer les services religieux protestants et de faire parvenir de la littérature protestante à ses abonnés; La Lanterne d'Arthur Buies faisait l'éloge du protestantisme naissant, "ennemi" du clergé, "futur vainqueur de la superstition".

Croyant d'ailleurs que le protestantisme engendrait les mêmes erreurs que le libéralisme, le clergé le condamnait en des termes souvent semblables. Quelle différence en effet entre la condamnation du journal libéral, "qui prétend, entr'autres choses, être libre dans ses opinions religieuses et politiques" prononcée par Mgr Bourget en $1858^{3}$, et l'année suivante, ce prône du curé de Notre-Dame de Québec réprimandant ses fidèles qui continuaient de recevoir la littérature des colporteurs protestants: "Quand sera-t-on assez catholique pour se laisser guider par les autorités ecclésiastiques? Ne voit-on pas que ce prétexte de juger par soi-même est déjà un penchant vers le protestantisme? Le principe protestant, c'est le jugement privé qui interprète tout et rejette toute autre autorité que sa propre lumière." 4

Raison de plus pour justifier contre la propagande protestante l'opposition systématique et organisée qui commença à la

2 Joseph de Maistre, Du Pape (18e ed., Lyon-Paris, 1862), 474, cité par Y.-M.-J. Congar, "L'ecclésiologie de la révolution française au concile du Vatican, sous le signe de l'affirmation de l'autorité", in L'ecclésiologie au XIXe siècle (Paris, 1960), 79.

3 Cité par Philippe Sylvain, "Libéralisme et ultramontanisme au Canada français: affrontement idéologique et doctrinal (1858-1865)", in Le Bouclier d'Achille. Regards sur le Canada de l'ère victorienne (Toronto, 1968), 226.

4 Archives de la paroisse N.-D. de Québec (APN-DQ), Cahier de prônes (CP), 25 mars 1859. 
fin de 1839 avec la fondation des Mélanges Religieux et les débuts de la série de retraites paroissiales prêchées par Mgr de ForbinJanson. Des habitants de Sorel, par exemple, témoignaient ainsi des fruits de leur retraite: “et si jamais des ministres d'erreur et d'impiété osaient se montrer parmi nous pour ravir notre foi, ce seul cri (Vive la croix) nous rendrait invincibles" ${ }^{5}$. Dès ce moment, une bonne part des manifestations de ce que les historiens ont appelé le "réveil religieux" et qui tendaient à raviver la foi et la pratique religieuses, apparaissaient aussi comme des réactions anti-protestantes. Ainsi en fut-il, en partie du moins, de l'objectif de Mgr Bourget lors de son premier voyage en Europe, en 1841 . Il voulait recruter des communautés religieuses afin de fonder des écoles, desservir les terres de colonisation, prêcher les retraites populaires et s'occuper de soins hospitaliers et de charité publique ${ }^{6}$. Même volonté de combattre la propagande protestante lorsque l'assemblée du clergé, réunie pour la fondation des $M e ́-$ langes Religieux, décida de faire publier des ouvrages de controverse, et lorsque, en 1840, l'imprimeur Perrault lança la collection "Bibliothèque Catholique du Canada" ", lorsque aussi, deux ans plus tard, le curé de Notre-Dame de Québec fonda la Société des Bons Livres et organisa une bibliothèque paroissiale ${ }^{8}$. C'est aussi pour empêcher la circulation de "bibles falsifiées" qu'en 1846, le curé Charles-François Baillargeon s'imposa de traduire et de commenter le Nouveau Testament ${ }^{9}$. Même objectif quand $\mathrm{Mgr}$ Bourget refusa de participer à la construction d'une "maison d'industrie" à Montréal ${ }^{10}$ et quand il réclama de l'archevêque de Québec la refonte du petit catéchisme ${ }^{11}$. Et encore, ce ne sont là que quelques-unes des manifestations qui prouvent à l'évidence

5 Mélanges Religieux, II : 112, cité par L. Pouliot, op. cit., 51.

6 L. Pouliot, op. cit., 78-79.

7 Ibid., 15-16.

8 APN-DQ, CP, 17 avril 1842. Deux ans plus tard, les Sulpiciens fondent l'Oeuvre des bons livres à Montréal et en 1850, les évêques instituent cette œuvre dans chaque paroisse, précisant dans leur circulaire défendre la lecture de bibles falsifiées ou publiées sans l'approbation des supérieurs ecclésiastiques (MEM, II: 116).

9 APN-DQ, CP, 4 juin 1846.

10 Ordre, 24 janvier 1860. Archives de la Chancellerie de l'Archevêché de Montréal (ACAM), RLB 13, Bourget à R. Bellemare, 9 novembre 1863. Le conseil municipal de Montréal discutait de l'opportunité de faire bâtir un édifice pour héberger les pauvres. Mgr Bourget s'y objecta craignant, entre autres choses, pour la foi des catholiques dans cette institution forcément multi-confessionnelle.

11 ACAM, RCD, 107, 9 mars 1865, "Résumé des observations du clergé de Montréal sur le catéchisme". Parmi de nombreux autres arguments, Mgr Bourget notait qu'il n'était pas "assez dogmatique pour des enfants qui vivent dans un pays protestant". 
que les réalisations protestantes aiguillonnaient le dynamisme de l'Eglise catholique.

Mais qu'en est-il justement de l'ampleur de ce mouvement de protestantisation? Quand a-t-il débuté? Quels en furent les promoteurs? En quelles circonstances? Pour quels motifs? Ce sont les questions qui m'ont guidé dans cette recherche sur le protestantisme canadien-français entre 1800 et 1840 .

\section{Le prosélytisme protestant au début du XIXe siècle}

Vers 1800, après la mort des trois pasteurs anglicans de langue française que l'administration britannique avait placés dans les villes de Montréal, Trois-Rivières et Québec ${ }^{12}$, - leurs conquêtes évangéliques furent très rares - l'Eglise anglicane délaissa ses efforts d'évangélisation des Canadiens français. Elle préféra compter sur l'administration coloniale pour tenter de se faire reconnaître dans les faits comme église établie, reléguer ainsi les autres dénominations religieuses au rang des religions tolérées en Grande-Bretagne et pour angliciser, puis protestantiser les Canadiens français au moyen du système d'éducation.

La cessation des activités missionnaires anglicanes auprès des Canadiens français, au moment où la plupart des autres dénominations religieuses réformées commençaient à s'y intéresser, donna lieu à des explications variées, autant de la part des anglicans eux-mêmes que des sectes opposées et plus récemment des historiens. Aucune cependant ne me semble, à elle seule, expliquer la situation. La faiblesse numérique du clergé anglican et l'inorganisation de l'Eglise sont, par ordre d'importance, les raisons les plus souvent invoquées, Charles James Stewart, qui remplaça Jacob Mountain à la direction du diocèse de Québec de 1822 à 1837, avait coutume de dire: "I am not prepared to attack them." Il préférait rester sur la défensive en songeant aux difficultés que son clergé éprouvait à desservir convenablement les anglicans et à contrebalancer les influences des sectes rivales ${ }^{13}$. A plus forte raison Mountain, à qui avait plus tôt incombé la charge d'organiser le diocèse de Québec, de fonder des paroisses, de recruter et de former des ministres, avait été encore moins bien préparé pour mener une telle attaque. En 1812, après 19 ans de

12 David Chabrand Delisle à Montréal de 1766 à sa mort en 1794, le

Suisse François de Montmollin à Québec de 1768 à 1803 et Jean-Baptiste-

Noël Vessyère, un ancien récollet, à Trois-Rivières de 1768 à 1800 .

13 Annals of the Colonial Church (Québec, 1849), cité par Arthur Maheux, "Le problème protestant", in Rapport de la Société canadienne d'histoire de l'Eglise catholique (RSCHEC) (1939-1940): 45. 
travail, il n'avait pas plus de 8 pasteurs dans le Bas-Canada ${ }^{14}$. De plus, autre explication, le clergé anglican très proche de l'administration britannique ne voyait pas l'avantage de soulever les catholiques contre eux en pratiquant un prosélytisme agressif dont le résultat le plus probant, croyait-il, serait de nuire à la cause anglicane ${ }^{15}$ en envenimant les relations entre les deux groupes ethniques, plus particulièrement à cette époque où le nationalisme exacerbé des Canadiens français embrassait à la fois les valeurs religieuses, ethniques et linguistiques. Enfin, le caractère aristocratique du clergé anglican, son militantisme antirépublicain, sa participation presque exclusive aux privilèges de l'Etat, sa puissance politique et économique, son degré supérieur d'éducation comparativement aux autres sectes réformées et son inaptitude à se faire entendre des petites gens, humbles et frustres, des terres de colonisation, lui faisaient souvent préférer le confort des villes et des paroisses bien établies aux durs labeurs de la vie missionnaire ${ }^{16}$.

$\mathrm{Au}$ début du XIXe siècle, le travail évangélique des sectes dissidentes, des méthodistes surtout, leur gagna de nouveaux adeptes; avec l'émigration, ils accrurent rapidement leurs effectifs, étendant parallèlement leurs activités aux Canadiens français. Dès 1803, Jacob Mountain note, à regret, que des "prédicateurs itinérants et improvisés s'abattent sur la province" "17. Ils distribuent des bibles et organisent des réunions de prières. Mais le succès auprès des catholiques de langue française est presque nul. Robert Baird, un Américain fort zélé pour la propagation de l'évangile, analysant la faiblesse de ces résultats, trente ans plus tard, en recherchait les causes, à tort ou à raison, dans l'hostilité des Canadiens français à l'égard des Américains et des Anglais. "Il fallait, soutenait-il, des ouvriers parlant la langue des Canadiens et [ne] leur inspirant aucune défiance nationale ${ }^{18 "}$.

Durant ces années, seuls quelques rares missionnaires de langue française œuvrèrent au Bas-Canada. Deux d'entre eux, dont l'historiographie n'a retenu ni les noms ni les activités, s'y

14 Jean-Pierre Wallot, Un Québec qui bougeait, trame socio-politique au tournant du XIXe siècle (Boréal Express, 1973), 196.

${ }_{15} \mathrm{~A}$. Maheux, op. cit., 45. John Irwin Cooper, The blessed communion; The origins and history of the Diocese of Montreal, 1760-1960 (Montréal $1960), 50$.

16 Une partie de cette argumentation a été puisée dans Donald Creighton, Dominion of the North (new edition, 1957), 218.

17 J. Hare et J.-P. Wallot, Les imprimés dans le Bas-Canada, 59-60.

18 Rapport de la Société des Missions évangéliques de Lausanne (RSMEL), (17 août 1842), 41. 
seraient rendus avant 1795 pour se retirer peu de temps après du côté de Niagara ${ }^{19}$. Puis en 1815 , la British Wesleyan Conference délégua à Québec Jean de Putron, originaire de l'île Guernesey. Il passa ensuite à Montréal où il tenta de fonder une école qui fut tout de suite dénoncée par le clergé catholique. Quand il cessa son travail, vers 1821, les résultats étaient à peine perceptibles et la société wesleyenne ne lui trouva pas de successeur ${ }^{20}$. A Québec, en 1828, un nommé Jacques Reeves converti au protestantisme "par les incessantes sollicitudes, au dire de l'abbé L.-E. Bois, de quelques méthodistes suisses" nouvellement arrivés au pays, haranguait les passants sur les places publiques et distribuait des tracts. Il signa une brochure, Le Catholique Canadien, que Bois attribue plutôt à la plume de celui qui l'avait converti ${ }^{21}$. Ce sont les seuls cas connus durant cette période.

A part Reeves dont la carrière dura à peine deux ans, il ne semble pas que l'on ait réussi à recruter d'autres évangélistes ou colporteurs canadiens-français. L'explication fournie par la Montreal Bible Society en 1838, en réponse à une demande de la

19 Rev. John Campbell, "The Huguenots and Presbyterian missions in Quebec", in J. Castell Hopkins, ed., Canada, and Encyclopedia of the country, IV: 56.

20 Paul Villard, Up to the Light. The Story of the French protestantism in Canada (1928), 78. L'auteur note: "He also speaks of gathering on hundred children to form a school, but the priests denounced him and the school is abruptly closed."

21 Archives de l'Université du Québec à Trois-Rivières (AUQTR), fonds Nicolet. Bois, collectionneur du XIXe siècle à qui nous devons une grande partie de la documentation conservée dans ce fonds, écrivait en marge de la brochure Le Catholique canadien (s.l.n.d.) que Reeves cordonnier et marchand de cuir, originaire de Montréal, était venu s'établir à Québec où il maria une Canadienne française qui lui donna 5 enfants. L'un devint notaire et un autre étudia le droit. A la mort de sa femme, en 1826 , il avait déjà pris l'habitude de boire au point qu'il ruina complètement son commerce. Il fut alors pensionné par les évangélistes protestants arrivés en 1828 et se prépara ainsi à la prédication. De citoyen respectable qu'il était, sa conversion autant que sa passion pour l'alcool lui valurent le mépris de ses concitoyens "qui le sifflaient même parfois le maltraitaient". Il survécut deux ans à son apostasie. Bois qui l'a connu personnellement témoigne des dernières années de sa vie, tout en nous révélant une facette intéressante de sa perception des prédicateurs protestants: "Nous affirmons que cet homme d'une obésité, d'une tournure d'esprit assez biscornue, était à cette époque de sa vie (à l'âge d'environ 50 ans) incapable de saisir, encore moins d'expliquer les paradoxes et les sophistiques idées qui composent le code évangélique des ministres ambulants. D'ailleurs flétri qu'il était par le vin, énervé et incapable de se rendre compte de ses idées, nous n'avons nullement été surpris quand nous l'avons entendu débiter en style de ruelle, ou de rustre en goguette, des phrases décousues, entremêlées d'insultes à des concitoyens, d'interpellations sales et fétides aux passants. Méprisé de tout le monde, cette victime du charlatanisme des prédicants ambulants (...)" 
société mère, la British and Foreign Bible Society, s'applique sans aucun doute à la période antérieure: "It has been found impracticable to find suitable men for this office among the native canadians who speak the French language; as this class is in a very low condition generally, in regard to education and more so in regard to religion ${ }^{22}$." Du côté de la France et de la Suisse, avant 1830, le recrutement n'était pas plus aisé, car le "réveil religieux" qui avait aiguillonné le zèle des sectes dissidentes de l'Angleterre et de l'Amérique était encore trop récent dans ces pays pour produire des résultats immédiats.

\section{Réveil religieux en Suisse et intérêt pour les missions américaines}

Le terme "réveil religieux" évoque l'idée du renouvellement de la vie spirituelle, du passage d'une inaction relative à une action évangélique intense. Ce fut à peu près ce que connut la Suisse à partir de 1817. Mais s'en tenir à cette simple caricature tendrait à minimiser un phénomène qui provoqua au sein des Eglises officielles une remise en question des formes d'apostolats et des enseignements théologiques pour entraîner finalement un schisme et de longues années de persécution contre les dissidents ${ }^{23}$.

Amorcé vers 1810 chez quelques étudiants du séminaire de théologie de Genève, le "réveil" fut intensifié par le séjour en cette ville, en 1816-1817, de l'Ecossais Robert Haldane, ancien officier de la marine anglaise qui avait abandonné sa carrière pour se dévouer à l'évangélisation ${ }^{24}$. Ses enseignements furent l'occasion d'un violent conflit entre quelques étudiants et la Com-

22 Monthly extracts of the correspondance of the British and Foreign Bible Society (février 1839): 680. Reproduction d'une lettre du rev. J. Thompson datée du 30 déc. 1838.

23 Daniel Robert, Les Eglises réformées en France (1800-1830) (P.U.F., 1961) , 345-445, plus particulièrement aux chapitres VI à X, donne un excellent aperçu du réveil religieux des Eglises réformées de France, accordant aussi une importance particulière au mouvement genevois. Nous nous en sommes inspirés de très près. Sur le sujet on lira aussi avec profit l'ouvrage de Léon Maury, Le réveil religieux dans l'Eglise réformée à Genève et en France (1892), 2 vol.

24 Il avait fondé en Ecosse, avec son frère James, une "Société pour la propagation de l'Evangile" en marge de l'Eglise officielle. Il arriva à Genève en décembre 1816 pour se rendre ensuite en 1817 à Montauban. Revenu en Ecosse, il continuera de s'intéresser aux Eglises européennes. Haldane et d'autres Britanniques jouèrent un rôle important dans le regain de vie spirituelle que connurent la Suisse et la France. Lui ou son frère formera vers 1840, à Edinburg, un comité pour aider au financement et au recrutement des missionnaires de la French Canadian Missionary Society. 
pagnie des pasteurs de l'Eglise de Genève à qui ils reprochaient une interprétation rationaliste des Ecritures et des enseignements erronés concernant la grâce, la prédestination et la christologie. Refusant d'obéir à un règlement de l'Eglise interdisant de prêcher et d'émettre des opinions sur les questions théologiques controversées, ceux qu'on appellera les pasteurs "évangéliques" s'en séparèrent en 1817 , ouvrant la voie à un mouvement parallèle quelques années plus tard dans l'Eglise réformée officielle du canton de Vaud.

Le "réveil" consistait principalement en une volonté de retourner aux enseignements de l'Evangile en s'écartant de toutes autres pratiques religieuses non expressément prescrites. Les dissidents convertis portaient peu d'intérêt pour l'institution ecclésiale qu'ils définissaient d'ailleurs comme "l'assemblée des croyants". Leurs activités se caractérisèrent surtout par une recrudescence des discussions théologiques; par les rééditions ou publications de la Bible, de commentaires des Ecritures, de revues, d'études et d'opuscules édifiants et apologétiques; par la renaissance du prosélytisme qu'appuyaient les nombreuses fondations de sociétés bibliques et missionnaires; par l'introduction de nouvelles méthodes d'apostolat, dont le colportage, la prédication des pasteurs itinérants et les réunions de prières improvisées partout au sein des familles de néophytes ou dans les lieux les plus divers; enfin, par la reprise des polémiques avec les catholiques.

Malgré des efforts soutenus, les dissidents des Eglises officielles ne réussirent pas à faire l'unité autour de questions aussi importantes que la prédestination, "le caractère exact et les limites de l' "inspiration" des Saintes Ecritures" et d'autres points secondaires, tel le baptême des enfants ${ }^{25}$. Ils visaient cependant un objectif commun qui était, suivant le mot du pasteur Henri Olivier, "d'embrasser le monde de leur charité" ${ }^{26}$. Un champ d'évangélisation s'ouvrait devant eux: l'Europe, puis le monde.

Persécutés en Suisse et frappés d'interdit, les dissidents fournirent alors à la France ${ }^{27}$ le personnel nécessaire pour donner un élan au "réveil" qui s'y amorçait. Leur préoccupation pour les missions américaines apparut un peu plus tardivement. Ils y

25 D. Robert, op. cit., 374-75. Haldane était personnellement défavorable au baptême des petits enfants (355, note 1$)$.

26 Rapport de la Société des Missions évangéliques de Lausanne (11 août 1841) : 33 .

27 Le "réveil" en France ne provoqua pas de schisme. 
furent intéressés par Haldane et diverses sociétés bibliques britanniques avec lesquelles ils entretinrent des échanges constants qui furent causes d'émulation réciproque.

Le premier missionnaire suisse à venir au Canada, Isaac Cloux, était originaire du Canton de Vaud et appartenait à la Société des missions évangéliques de Lausanne, fondée en 1828 , dans le but principalement de répandre la connaissance de l'évangile chez les peuples non chrétiens. Après avoir débuté dans le colportage biblique et entretenant déjà l'idée d'œuvrer dans les missions américaines, Cloux entra à l'Institut missionnaire nouvellement fondé par la société vaudoise. Plus apte au travail d'évangélisation qu'aux études théoriques (il était menuisier), il y resta à peine une année. Il manifesta dès le début son intention de partir pour le Canada. Les membres de la Société écrivirent alors à un compatriote suisse de Québec, "M. le ministre Blanchet" ${ }^{28}$, pour qu'il fasse les arrangements en vue de son arrivée prochaine au Bas-Canada. Leur lettre fut remise à $\mathbf{M}$. Plenderleath ${ }^{29}$ qui, dans la correspondance suisse, est présenté comme fonctionnaire au "département indien à Montréal". L'évêque de Québec Stewart et son archidiacre Jehosaphat Mountain furent tous deux mis au courant et donnèrent l'assurance qu'Isaac Cloux "trouverait assez facilement les moyens de gagner sa vie en exerçant sa vocation de missionnaire" ${ }^{30}$. Plenderleath, se chargea de faire transmettre cette réponse à Lausanne par l'intermédiaire de Blanchet et obtint du gouverneur James Kempt une gratification annuelle de "20 louis" pour le nouveau missionnaire. Cette somme était déjà octroyée au missionnaire qui avait desservi le village indien de St-François, Peters Paul, à qui Cloux devait succéder. Son apostolat pourrait être orienté du côté de

${ }^{28}$ Aucun document canadien consulté ne fait mention de ce missionnaire. Il se peut qu'il soit un de ceux dont parle l'abbé Bois et qui fut à l'origine de la conversion de Jacques Reeves. Par ailleurs, John Campbell, op. cit., 56, laisse entendre que la British Reformation Society, aurait délégué à Québec, vers 1830, à la demande du capitaine Anderson, dont je reparlerai plus loin, un missionnaire de langue française sans plus de renseignements à son sujet. En fait, l'influence des sociétés bibliques et missionnaires étrangères au Canada est aussi mal connue que l'histoire du protestantisme au Bas-Canada.

29 Ce Plenderleath est sans doute William Plenderleath Christie, un anglican zélé qui participera à la fondation de la French Canadian Missionary Society. Grand propriétaire terrien dans la région d'Iberville, il s'intéresse au moins depuis 1828 à l'évangélisation des Canadiens français et des Indiens. Il entretient à ce sujet une correspondance abondante avec l'évêque anglican de Québec qu'il dotera de deux églises à cette fin. l'une à Sabrevois, sur ses terres, l'autre à Montréal. Voir à ce sujet J. I. Cooper, op. cit., 37-38, 50ss.

30 RSMEL (1830) : 40 à 43. 
l'enseignement aux Indiens en anglais ou en français. Cependant Plenderleath ne cachait pas ses préférences: "Je le considère [...] comme étant [...] en état de commencer en anglais avec les Indiens ${ }^{31}$." Sa lettre pouvait paraître d'autant plus encourageante pour la Société vaudoise qu'il leur demandait d'envoyer deux missionnaires plutôt qu'un.

Arrivés à New-York à la fin de 1830, Isaac Cloux apprit que la rénumération proposée par le gouvernement James Kempt n'avait pas été maintenue par son successeur Lord Aylmer ${ }^{32}$. L'évêque de Québec et Blanchet lui recommandèrent de se rendre à York. Entre-temps, il fit part de ses déboires à la Société de Lausanne qui lui répondit de passer l'hiver à New-York. C'est là qu'il connut personnellement l'évêque de Québec. Leur première rencontre fut chaleureuse; mais, à la seconde, Cloux exposa quelques aspects de ses croyances religieuses; l'évêque de Québec stupéfait lui retira immédiatement les recommandations qu'il lui avait déjà accordées ${ }^{33}$. Se référant de nouveau à Plenderleath, il obtint une mission chez les Indiens de la région de Kingston. Il y resta peu de temps, pressé d'abandonner cette tâche difficile qui risquait de ruiner sa santé. Il trouvait d'ailleurs pénible d'enseigner à des enfants indisciplinés pour qui la réprimande un peu sévère "était un moyen de les faire fuir de l'école". "Ce qui n'était pas moins pénible encore, ajoutait-il, c'était de demeurer une grande partie de la journée renfermé avec eux dans la chambre. Ils étaient enduits de graisse et répandaient une odeur si désagréable" qu'il devait sortir pour respirer librement. Il préféra se rendre à Montréal où on tenta en vain de lui donner un poste de maître d'école chez les Canadiens français des environs de l'Acadie. Isaac Cloux retourna à Lausanne au début de $1832{ }^{34}$.

31 Ibid.: 41.

32 A quoi attribuer ce changement d'attitude? Suivant les raisons invoquées devant Cloux "des garanties assurées aux catholiques romains lors de la session du Canada, entravaient de mille manières toute institution évangélique". (Feuille religieuse du Canton de Vaud (FR) 10 juillet 1831, 325). En fait, en 1830 , le "Département des Sauvages" avait été transféré de l'administration militaire à l'administration civile, et le territoire avait été divisé en deux sections. Les missions indiennes subventionnées par le gouvernement dans le Bas-Canada étaient réservées aux catholiques, celles du Haut-Canada aux protestants. (Lionel Groulx, "La situation religieuse au Canada français vers 1840", in RSCHEC (1941-42) : 61).

33 RSMEL (1831) : 30.

34 RSMEL ( 7 novembre 1832): 38 . Tous nos renseignements concernant la mission de Cloux en Amérique ont été puisés dans la Feuille religieuse du Canton de Vaud, et dans les Rapports de la Société des missions évangéliques de Lausanne de 1830, 1831 et 1832. 
La société vaudoise avait déjà fait connaître dans une circulaire parue dans la Feuille religieuse de janvier 1831, son regret de ne pas avoir de missionnaires disponibles pour les missions canadiennes où plusieurs d'entre eux pourraient être placés. Elle signalait un peu plus tard que les Suisses de langue française étaient particulièrement désignés pour les missions indiennes du Canada, faisant valoir que les Américains en fournissaient peu et que seuls des francophones pouvaient “contrebalancer l'influence $d u$ prêtre catholique sur les Indiens, influence qui [était] due en grande partie à la langue française qu'ils parl[aient] et que les Indiens aim [aient] entendre parce qu'ils y attach [aient] des souvenirs ${ }^{35}$ ".

De leur côté, les Français, au dire du doyen de la faculté de Montauban à qui une société britannique demandait des missionnaires, pourraient être l'objet d'un certain ressentiment national en œuvrant "dans un pays autrefois français et actuellement sous la domination anglaise" ${ }^{36}$. D'un avis assez général, il y avait donc tout avantage à ce que les missionnaires fussent d'origine suisse. Malgré cela, le retour prématuré de Cloux en 1832, son incapacité à fonder une station permanente auprès des Indiens et son refus d'œuvrer à l'Acadie que motivaient autant les raisons de santé que le respect du premier objectif de sa société, l'évangélisation des païens, risquaient de mettre fin aux préoccupations vaudoises pour les missions canadiennes. Le comité délibéra et demanda conseil à la London Missionary Society ${ }^{37}$ qui avait d'abord inspiré leur action missionnaire. Le comité avait d'ailleurs conscience de demander l'avis d'une société qui possédait une vaste expérience dans le domaine des missions américaines. La réponse vint: le Canada. ${ }^{38}$

\section{La Société des Missions évangéliques de Lausanne au Canada}

Le comité de Lausanne s'adressa de nouveau à Plenderleath pour s'enquérir des besoins du Canada. Celui-ci leur fit répondre par le capitaine (plus tard général) Anderson, particulièrement préoccupé à ce moment par l'évangélisation des catholiques ${ }^{39}$ :

35 RSMEL (1831) : 39.

36 RSMEL (1831) : 39.

37 Société fondée sur une base non-ecclésiastique et "interdénominationnelle" en 1795 .

38 RSMEL ( 7 novembre 1832 ) : 43-44.

39 Cet Anderson qui d'après la correspondance suisse a remplacé Plenderleath comme fonctionnaire auprès des Indiens est sans doute $T$. G. Anderson "superintendent of Indian Affairs". 
Un champ de travail particulièrement intéressant semble se présenter soit dans le Haut, soit dans le Bas-Canada. Le Papisme a un champ étendu dans la province inférieure, et l'indifférence pour toute religion domine déplorablement dans la supérieure [...] Nous concevons que deux hommes fidèles, d'un esprit simple, seraient bien placés, l'un dans le voisinage immédiat de Québec, où il y a une tribu d'Indiens qui parlent français, et l'autre à l'Acadie, à quelques milles de Montréal [...] au milieu d'un certain nombre de canadiens tous préparés à le recevoir.

[...] J'ai principalement fait mention des stations parmi les Indiens me souvenant que votre société borne ses travaux aux payens. Mais dans bien des endroits la population blanche est dans d'aussi grands ténèbres que les payens [...] et je serais réjoui si nous pouvions servir d'humbles instruments pour vous engager à envoyer des missionnaires exprès pour prêcher l'évangile parmi la population papiste du Canada. [. . .] et je crois, d'après leur langage dans bien des endroits qu'ils ne s'inquièteraient pas des menaces de leurs prêtres qui voudraient les empêcher d'entendre les hommes de Dieu [...] pourvu que le missionnaire qui s'adresserait à eux parlât couramment le français ${ }^{40}$.

Cette lettre lue devant les membres de la Société suscita ce commentaire, sans qu'il ne fut fait mention de réorienter les objectifs: "Lorsque nous aurons de nouveau quelqu'un à envoyer, ce qui sera, à vue humaine, dans environ un an et demi, nous nous croirons bien probablement appelés à y diriger nos élèves ${ }^{41 . "}$ En attendant, il fallait les préparer adéquatement. Les règlements de l'Institut missionnaire, fondé en 1829, étaient à ce sujet très sévères. Seuls les candidats âgés de 16 ans pouvaient être admis après une enquête approfondie sur la sincérité de leur vocation de missionnaire. Les études duraient quatre années; durant les premiers six mois, les étudiants subissaient des épreuves dont le succès dépendait du jugement de la majorité des membres du Comité de direction. Outre une évaluation des aptitudes intellectuelles du candidat, l'épreuve principale consistait en un examen des qualités morales fait individuellement par chacun des membres du comité ${ }^{42}$.

Mais une formation en apparence si prometteuse ne fournit pas les résultats attendus. En 1836, l'Institut missionnaire sus-

40 RSMEL ( 7 novembre 1832) : 43-44. Nos connaissances actuelles ne nous permettent pas de critiquer ce témoignage sur les dispositions des habitants de l'Acadie à l'endroit du protestantisme.

41 Ibid.: 47-48.

42 Ibid., (4 novembre 1829) : 30-32. 
pendit ses activités après que les étudiants, pour des causes diverses ${ }^{43}$, eurent quitté un à un l'établissement. Deux d'entre eux, Daniel Gavin et Samuel Dentan, interrompirent leurs études en 1834 pour accompagner Henri Olivier et sa femme dans les missions canadiennes.

Henri Olivier était un membre en vue de la Société des Missions évangéliques de Lausanne. Exilé à Paris avec son frère François, il l'avait assisté quelque temps à la direction d'un centre de formation de pasteurs, soutenu par Robert Haldane de 1824-25 à 1832. Cet institut avait au tout début accueilli des étudiants de Lausanne dont les études avaient été interrompues par les persécutions ${ }^{44}$. Sa décision de se consacrer aux missions canadiennes en surprit plusieurs car, suivant les descriptions faites par Cloux des difficultés de servir chez les Indiens, il n'avait certes pas la jeunesse et les qualités physiques nécessaires pour affronter ces labeurs. Du reste, il semble bien que ce fut pour cette raison qu'à son départ du Havre, le 15 août 1834, il ait laissé entendre que "son dessein était de faire l'œuvre que le Seigneur lui montrerait quand il serait arrivé, sans exclure les Payens, près desquels il espérait pouvoir trouver une station convenable pour agir sur eux ${ }^{45}$ ".

Sitôt arrivés à Montréal ${ }^{46}$, les trois missionnaires furent sollicités d'œuvrer auprès des Canadiens français par le pasteur presbytérien Perkins et trois autres collègues de dénominations religieuses différentes réunis pour la circonstance. Olivier c'était prévisible - accepta une classe d'enseignement dans la petite église méthodiste et envoya personnellement deux lettres au comité pour plaider en faveur d'une modification des objectifs. Puis quelques Ecossais apprenant sa présence au Bas-Canada sollicitèrent à leur tour la Société d'y consacrer son œuvre. Rien n'y fit. Dentan reçut la recommandation d'abandonner l'école de l'Acadie qu'il dirigeait depuis quelque temps pour rejoindre Gavin dans les missions du Haut-Canada. Pourtant, les débuts étaient encourageants; il enseignait à vingt-six enfants et le nouveau poste qu'on lui offrait quelques milles plus loin lui en

43 Parmi les causes du départ des étudiants, il y avait le désaccord avec le règlement de l'institution (RSMEL (1836): 9). Lors de la fondation, certains avaient objecté que l'institution ne pourrait marcher en paix car elle réunissait des élèves appartenant à des Eglises différentes (RSMEL (1830) : $33)$. Nous ne savons pas si ce fut une cause du départ des étudiants.

44 D. Robert, op. cit., 365.

45 RSMEL (11 juin 1835) : 26.

46 Les Olivier étaient aussi accompagnés d'un domestique. Ils arrivèrent à Montréal le 31 octobre 1834 . 
fournirait une soixantaine ${ }^{47}$. La Société de Lausanne avait répondu qu'elle "ne mettait aucun obstacle à ce qu'il se formât une station dans le Bas-Canada, parmi les catholiques, pourvu que l'on ne perdît pas de vue les Paiens, objet propre de la Société". ${ }^{48}$

Contrairement à Dentan et Gavin, qui se conformèrent aux directives de Lausanne, Olivier fut obligé d'annoncer qu'il ne pouvait plus se considérer comme employé de la Société, car l'état de sa santé ne lui permettait pas de "se livrer à l'apprentissage d'une nouvelle langue". Mais même si sa mission n'était plus financée par la société vaudoise ${ }^{49}$ et que tous liens officiels étaient rompus, Olivier et sa femme continuèrent à recevoir des encouragements de Lausanne et à y envoyer une correspondance régulière que publiaient à l'occasion les rapports des diverses sociétés missionnaires suisses et la Feuille religieuse $d u$ Canton de Vaud. Ces relations de missions ajoutées aux lettres personnelles d'Olivier adressées à des amis contribuèrent à entretenir l'intérêt des Suisses pour les missions canadiennes-françaises et à y gagner de nouveaux missionnaires: en octobre 1835, madame Henriette Feller, une amie d'Henri Olivier, et Louis Roussy arrivèrent à Montréal.

L'expérience missionnaire auprès d'un peuple jugé au premier abord "d'ignorants et de supersticieux" s'avéra des plus pénibles. Madame Olivier qui venait d'ouvrir une petite école pour les jeunes filles de Montréal jugeait que "sans être cramponné au Seigneur", l'œuvre était impossible. "Si vous la [ville de Montréal] voyiez de près, disait-elle, si vous entendiez les discours de la partie de la population qui est le champ spécial que mon mari doit essayer de défricher, je crois que vous pourriez comprendre que même le cœur naturel pousserait plutôt vers un terrain tout neuf, vers des hommes qui n'ont que l'ignorance naturelle, et non pas celle que les prêtres ont inculquée à ce peuple et dont ils l'entourent comme un triple filet. Il est d'une grossièreté, d'une hardiesse, d'une ignorance des plus repoussantes. L'usage des liqueurs abrutit encore beaucoup plus que notre vin [...] ${ }^{50}$ ". D'un autre côté, l'apostolat d'Olivier se butta continuellement à l'influence du clergé. L'école établie dans l'église méthodiste n'avait pas, à son dire, un grand succès car sitôt qu'il s'opposait ouvertement au clergé, les étudiants la désertaient. Il fut même obligé de changer de local; l'explication vaut d'être

47 RSMEL (11 juin 1835) : 44.

48 Ibid.: 33.

49 RSMEL (11 juin 1835) :34-35.

50 FR (25 janvier 1835): 59 . 
retenue: "Au moins, y a-t-il maintenant plusieurs Canadiens qui viennent volontiers nous voir, mais qui n'oseraient pas aller dans la salle d'école, parce qu'elle fait partie d'un bâtiment consacré à un culte protestant ${ }^{51}$." En somme, les résultats de la mission d'Olivier étaient décourageants. S'il trouvait "quelques sujets de joie" à occuper ses soirées "à enseigner, à lire, écrire et chiffrer à des jeunes qui n'ont plus la facilité de l'enfance ${ }^{52 "}$ ", il ne réussit que trois conversions en quinze mois de travail ${ }^{53}$. Aussi décida-t-il au printemps 1836 de rentrer au pays, invoquant des raisons de santé et son incapacité de s'adapter au climat.

\section{Les débuts de la station de Grande-Ligne}

Le départ des Olivier laissa Madame Feller et Louis Roussy dans une situation précaire; les débuts de leurs missions n'étaient pas plus fructueux; Roussy qui avait accepté la succession de Dentan à l'école de l'Acadie réussit quelque temps à cacher au clergé la vraie nature de son travail. Après deux mois, celui-ci le força à quitter. Pour la même raison, Mme Feller abandonna sa classe à Montréal. Ils se tournèrent du côté de St-Jean où les méthodistes mirent une chapelle à leur disposition; même échec: "Priestly opposition could not be overcome", écrivait Roussy. L'école n'attirait personne et le colportage était de plus en plus difficile. On leur fermait les portes des maisons, les brutalisait parfois, et un jour, on tenta le tuer le cheval de Roussy. Bref, incapables de se fixer, ils abandonnèrent trois classes en une année et demie de travail ${ }^{54}$. C'est à ce moment qu'ils convertirent madame Lore de l'Acadie. Agée d'environ soixante (60) ans, elle avait déjà pratiqué le protestantisme aux Etats-Unis, son pays natal, avant d'immigrer au Canada et de se marier à un catholique. Elle présenta Roussy aux nombreux membres de sa famille et particulièrement à la famille de son gendre, les Lévesque qui habitaient Grande-Ligne, à quelques milles au sud de St-Jean. Les évangélistes allèrent s'y établir à l'automne 1836 et formèrent une petite communauté constituée presque exclusivement, au début, de la progéniture de Madame Lore ${ }^{55}$.

51 RSMEL (11 juin 1835) : 36.

52 Ibid.: 37

53 R.-P. Duclos, Histoire du protestantisme français au Canada et aux Etats-Unis (Montréal, 1913), 113.

54 Sur les premières années d'apostolat de Feller et Roussy, consulter

J. M. Cramp, A memoir of Madame Feller. With an account of the origin and progress of the Grande Ligne Mission (Londres, s.d.), 78-110.

55 FR (10 mars 1844). Le choix de Grande-Ligne, comme nous le verrons plus loin, ne se justifiait pas seulement par la présence des descen- 
Ces deux premières années de travail furent riches en enseignements pour des missionnaires qui ignoraient tout de la mentalité et des coutumes des Canadiens français. Par exemple, le décès de Madame Lore, en 1836, fut l'occasion d'observer les pressions ultimes faites au chevet de la malade, non pas par le curé avec qui elle n'avait plus de relations depuis un certain temps, mais par un ami, son émissaire, qui n'eut pas de succès. Quelques membres catholiques de la famille s'étaient associés pour la même fin, sans plus de résultat. Ils purent constater combien sa conversion avait perturbé son entourage; son refus de revenir au catholicisme lui valut l'ostracisme de la communauté paroissiale. Les paroissiens craignirent d'abord, puis souhaitèrent - car c'était une grande disgrâce - qu'elle fut enterrée dans un champ derrière sa ferme. En fait, elle fut inhumée dans le cimetière protestant de St-Jean, et au passage du cortège, les villageois attroupés ne virent que des Anglais. Rares furent les Canadiens français qui vinrent s'y associer ${ }^{58}$. Leçon à retenir pour quiconque s'avisait de se convertir au protestantisme.

La résistance des mentalités, observèrent les évangélistes, constituaient un puissant obstacle à leurs conquêtes. Rieul Duclos, un converti de cette époque, en trouvait l'explication dans l'influence d'un clergé autoritaire qui ne favorisait pas l'instruction du peuple ${ }^{57}$. Mme Feller ne pensait pas différemment et, en dépit de ses déboires, elle conservait la conviction que l'évangélisation des Canadiens français devait commencer "par l'école" 58. Mais encore fallait-il trouver une population assez indépendante des exhortations du clergé. Par exemple, son école gratuite à St-Jean avait été désertée après les remontrances du curé ${ }^{59}$. La ville leur était pratiquement fermée; dans les villages, il n'était pas facile de tromper la vigilance du curé bien informé

dants de madame Lore. Cette présence amicale était cependant fondamentale. L'exemple de Grande-Ligne s'appliquerait-il aux paroisses de St-Jean et de l'Acadie où œuvrèrent les évangélistes? Nous ne saurions conclure avec précision. Peut-être y avaient-ils en ces lieux des protecteurs anglais, des familles canadiennes-françaises sympathiques au protestantisme, ou encore des paroissiens en brouille avec le curé. Notons qu'avant 1839, c'est cette région qui, à notre connaissance, est considérée comme la plus prometteuse par les évangélistes.

56 J. M. Cramp, op. cit., 93.

57 R.-P. Duclos, op. cit., 114.

58 FR (13 janvier 1839).

59 Cramp, op. cit., 89. 
des faits et gestes de chacun de ses paroissiens ${ }^{60}$. Restaient les terres de colonisation visitées irrégulièrement par le clergé fort occupé dans les paroisses et les séminaires.

Grande-Ligne, où s'établirent Mme Feller et Roussy ${ }^{61}$ en septembre 1836, était précisément ce type de territoire où l'on ne faisait que commencer le défrichement. C'était "une longue route de chaque côté de laquelle étaient échelonnées les maisons des fermiers et partageant par une ligne droite longue de plusieurs lieues des concessions de terrains ${ }^{62}$ "'. Il n'y avait évidemment pas encore d'école et cela fournit aux évangélistes un premier moyen d'apostolat. Mais la vie dure du défricheur et les échanges de services occasionnés par l'économie de subsistance qui est souvent la règle dans ces territoires difficiles d'accès provoquaient l'entraide et les bonnes relations de voisinage. Dans ce système communautaire, les évangélistes s'intégrèrent d'abord par l'intermédiaire de la famille Lévesque, puis par les parents des élèves auxquels ils s'efforçaient de rendre service. Ayant remarqué que les fermiers ne cultivaient qu'une moitié de leurs terres, laissant le reste en pâturage, Mme Feller convainquit un ami anglais du Bas-Canada de leur prêter l'argent nécessaire à l'achat de graines de semence. Elle pouvait ainsi écrire en 1838: "We have persuaded our people this year [...] to plough and sow all their land, the meadows only excepted, which produce abundant crops of hay. This is an innovation, and is regarded as a remarkable event. ${ }^{68}$ "

Le soir, quelques membres de la petite communauté se réunissaient chez les évangélistes et assistaient à la lecture et à la rédaction de leur courrier personnel. La soirée se terminait toujours par la méditation d'une page de la Bible. Mme Feller accourait aussi au chevet des malades et épargnait souvent au patient, par ses soins et médicaments personnels, les frais d'un médecin ${ }^{64}$. Elle écrivit: "Une femme d'une méchanceté dont il n'y a pas, je crois, beaucoup d'exemples ailleurs [...], disait [...]

60 Duclos, op. cit., 14, écrit: "dans les villages surtout, constamment parcourus par $M$. le curé, qui en arpente les rues, le bréviaire à la main, saluant avec dignité les parents, caressant les enfants, l'œuvre est vraiment difficile".

${ }_{61}^{6}$ Le frère de Louis Roussy, Henri, vint rejoindre les évangélistes à Grande-Ligne en 1837 (peut-être au printemps). Il rendit quelques services à la communauté. A l'automne 1838, il tient un commerce à Napierville.

62 Ibid., 117.

63 J. M. Cramp, op. cit., 115, 125-126.

64 Le père de Mme Feller avait été directeur de l'hôpital cantonal de Lausanne. C'est dans ce milieu qu'elle acquerra quelques connaissances médicales. 
qu'elle aimerait mieux mourir que de me demander ou de me rendre un service. Dans le courant de l'hiver, j'eus occasion de la voir avec un de ses enfants qui était faible et qui me paraissait demander des soins bien prompts [...] elle perdit toute espérance de le conserver; alors elle vint me supplier d'aller le voir et de lui être en aide [...] tous les remèdes ont produit le meilleur effet. L'enfant est très bien rétabli, et le père et la mère sont au nombre de nos amis [...]. La Parole de Dieu est dans leur maison, leurs enfants à mon école, et nous espérons voir cette famille entière soumise au Seigneur Jésus-Christ. ${ }^{\circ 5}$ "Ainsi se pratiquait l'apostolat évangélique à Grande-Ligne.

Un tel succès, pourtant bien minime, contrastait singulièrement avec les déboires des débuts. Pour autant, tout n'allait pas sans heurts. La visite du prêtre desservant, en janvier 1837, fit craindre la désertion des membres. Une fois ce moment passé, le travail d'évangélisation fut maintes fois entravé par des méchancetés et des vexations de toutes sortes. On disait de ceux qui acceptaient de recevoir les pasteurs qu'ils étaient à "moitié revirés" 66. Les brimades et les persécutions augmentèrent avec l'approche des journées insurrectionnelles de 1837. En octobre, la communauté comptait seize membres, dix autres Canadiens français étaient prêts à y entrer ${ }^{67}$. Mais au prix de quelles humiliations! Les nouveaux convertis étaient sujets "aux malices [des] voisins" qui ravageaient leur jardin et, fait plus grave, coupaient la crinière et la queue de leurs chevaux en sorte qu' 'ils ne pouv [aient] aller nulle part sans être aussitôt reconnus et exposés aux moqueries et aux insultes". ${ }^{68}$ "Il faut être, écrit Mme Feller, bien habitué à l'opprobre pour voyager avec eux au milieu d'un peuple ennemi." Puis, au début de novembre, la révolution qu'ils n'avaient pu prévoir tant "les Canadiens paraissaient si peu murs", les obligea à quitter le territoire canadien ${ }^{69}$.

A l'exception des néophytes évangélistes, presque tous les habitants de Grande-Ligne étaient patriotes. Au dire de Mme Feller, c'était "un des endroits du pays reputés les plus mauvais". Ceux qui n'entraient pas dans le mouvement, suivant son témoignage, étaient soumis aux pires menaces:

$65 \mathrm{FR}$ (11 novembre 1838): 530.

66 FR (11 novembre 1838): 530 .

67 FR (11 février 1838) : 90.

68 FR (11 février 1838): 91 .

69 FR (11 février 1838): 91-95. Les renseignements qui suivent ont été puisés dans une lettre de Madame Feller, datée du 18 novembre 1837, et reproduite dans ce numéro. 
Leurs expéditions avaient toujours lieu la nuit; ils se rendaient en masse de cent, deux cents, trois cents, et quelquefois bien davantage contre un seul individu; ils étaient tous masqués et munis de tous les instrumens imaginables pour faire un charivari complet. Ils commençaient par là, mêlant à leur musique infernale des cris, des imprécations, des abominations plus infernales encore. Ceux qui ne se rendaient pas immédiatement avaient leurs maisons assaillies de pierres et menacées de feu. (Ils portent des brandons allumés.) Il y en a eu dont les maisons ont été renversées, et chez lesquels tout a été brisé, pillé. Tout ce que je pourrais vous raconter de ces malheureux ne vous donnerait jamais une idée de ce qu'ils sont: il faut les avoir vus et entendus; et encore en les voyant et les entendant, j'avais peine à me persuader que ce fussent des hommes. ${ }^{70}$

On peut ainsi imaginer que le refus des convertis de participer à la rébellion aggrava aux yeux des patriotes une situation déjà passablement détériorée par leur conversion à la croyance de l'ennemi anglais. Du reste, tous savaient et amplifiaient au gré de leur imagination bien meublée par une campagne de dénigrement que des Anglais de Montréal pourvoyaient aux besoins financiers de la petite communauté. Ils percevaient donc l'œuvre des évangélistes suisses comme un moyen pris par les administrateurs britanniques pour "changer" non seulement "leurs mœurs mais aussi leur religion". " Devant Mme Feller, ils arguaient ne plus vouloir "souffrir dans leur endroit que des gens professant leur bonne religion et qui fussent bons patriotes comme eux", et l'accusaient d'être venu "troubler le pays en y apportant une religion nouvelle" ${ }^{72}$. C'est donc paradoxalement en partie par attachement à leur religion et au risque de l'excommunication que les insurgés s'arrogeaient le droit de forcer les convertis à choisir entre abjurer le protestantisme ou quitter le pays. $^{73}$

Leur départ fut fixé au $1^{\text {er }}$ novembre; environ cinquante Canadiens traversèrent les frontières pour aller s'établir à Champlain. Dans une même charrette étaient entassés dix enfants et deux femmes mal vêtus pour affronter les froids de l'automne:

70 FR (11 février 1838) : 92. Cramp, op. cit., 111.

71 Déposition d'un prévenu (Robillard), cité par F. Ouellet, "Les insurrections de 1837-38, un phénomène social", in Eléments d'Histoire sociale du Bas-Canada, 365-366.

72 FR (11 février 1838) : 93. Lettre de Mme Feller, 18 novembre 1837.

73 Epoque de confusion, d'écrire Ouellet (op. cit., 366 ) où l'anticléricalisme de certains ruraux ne peut être interprété comme le signe de leur rupture avec les valeurs religieuses traditionnelles. 
c'est la description pathétique faite par Mme Feller dans les journaux américains, anglais et suisses, qui leur valut les sympathies multiples du monde protestant et.. un financement plus aisé ${ }^{74}$; car l'exil ne mit pas fin à leur projet, au contraire, ils étaient bien décidés à revenir d'autant plus que les journées insurrectionnelles leur rapportaient l'espérance d'un plus grand succès. "Un des heureux contre-coups de cette guerre, écrivait Mme Feller, est d'avoir brisé le joug des prêtres; ils n'ont exercé aucune influence sur les rebelles, qu'ils essayaient de retenir par des menaces d'excommunication; mais personne n'en a tenu compte." 75

\section{Espoirs suscités par la rébellion et nécessité du prosélytisme}

La rébellion de 1837-38 marquait une étape importante de la progression du protestantisme au Canada français parce qu'elle apparaissait aux yeux des évangélistes anglais et suisses comme l'occasion de percer ce bouclier qu'était l'influence du clergé. Mme Feller le remarqua une première fois au moment de l'exode. De plus, les destructions et les dévastations consécutives à la rébellion ne seraient-elles pas causes de disettes et ne lui fourniraient-elles pas les moyens pour établir son influence et se faire accepter d'une majorité de la population au premier abord hostile? Par exemple, à leur retour de Champlain après deux mois d'exil, les évangélistes utilisèrent l'argent reçu de Suisse et des Etats-Unis pour acheter de la nourriture et des remèdes qui profitèrent à une cinquantaine de personnes ${ }^{76}$. Ils annoncèrent aussi publiquement devant les intéressés réunis pour la circonstance qu'ils retiraient leur poursuite en justice contre tous ceux qui avaient pillé les maisons des protestants et les avaient forcés à l'exil ${ }^{77}$. Mme Feller put alors écrire le 4 mai 1838: "En général, l'esprit du peuple est tellement changé envers nous, qu'il n'est, je crois, aucune maison de la Grande-Ligne dans laquelle je ne puisse entrer maintenant. On nous témoigne autant de respect et de confiance qu'on avait d'éloignement autrefois." " 78 Le nombre des membres de la communauté augmenta à soixante et quarante enfants assistaient à la classe. L'échec de la rébellion de 1838 fournit aussi à Mme Feller l'occasion de faire valoir son influence auprès d'amis anglais, dont Richard MacGinnis, chargé à Napier-

${ }^{74}$ Cramp, op. cit., 115. De la Suisse, elle reçut " 4000 francs (830 dollars)".

${ }_{75} \mathrm{~F}_{\mathrm{R}}$ (11 février 1838) : 95.

76 FR (11 novembre 1838). Lettre de Mme Feller, 4 mai 1838.

77 Ibid.

78 FR (11 novembre 1838). Lettre de Mme Feller, 4 mai 1838. 
ville, "de recevoir les dépositions et d'examiner les prévenus". Elle les pria d'abandonner les accusations: "Tous ceux, dit-elle, desquels j'ai pu rendre le témoignage qu'ils n'avaient pas suivi volontairement les chefs insurgés, sont à l'abri de tout châtiment." 79 Puis elle ajouta : "Il faut avoir vu la scène de mon retour pour s'en faire une juste idée. La maison était remplie de monde; et tous de s'écrier: "Quelles nouvelles?" La joie, la reconnaissance furent grandes; tous furent consolés [...] Dès lors nous voyons à notre culte des hommes que la Parole de Dieu faisait fuir précédemment." 80

Il était à prévoir que cette percée du protestantisme au gré d'une conjoncture matérielle et psychologique favorable ne résisterait pas à la riposte catholique des années 1840; mais, pour l'instant, elle provoquait de nombreux espoirs autant à GrandeLigne que dans le reste du Bas-Canada. Des missionnaires furent donc à nouveau demandés aux sociétés suisses et françaises ${ }^{81}$. De leur côté, les sociétés bibliques anglaises et les diverses dénominations protestantes redoublèrent d'activité, car tous avaient fait la même observation que Louis Roussy, reproduite dans les journaux suisses et américains: "Et ce n'est pas à la GrandeLigne seulement, c'est en général dans la contrée que l'influence du prêtre diminue. Depuis les derniers troubles surtout, les Canadiens ne craignent plus d'exprimer ouvertement les doutes dont à peine auparavant ils osaient parler en secret." 82 Ils pensaient comme Mme Feller: "Le temps est venu, le Canada est ouvert." 8s

Aux réunions de la Montreal Bible Society, à la fin de 1838, les membres des diverses dénominations religieuses présentes ne comptaient plus les heures passées à discuter des moyens à prendre pour évangéliser les Canadiens français: échanges de correspondance avec Londres sur ce sujet, recherches de colporteurs canadiens-français, pourparlers avec les sociétés suisses, toutes recherches qui tendaient à donner au prosélytisme protestant un caractère français. Les succès relatifs de Mme Feller à Grande-Ligne comparés aux difficultés des colporteurs de langue anglaise de se faire entendre dans les familles canadiennesfrançaises étaient certes une des raisons de cette réorientation.

79 FR (13 janvier 1839) : 24-25.

80 Ibid.

81 FR (11 novembre 1838 et 13 janvier 1839). Le 11 mars 1838, le rédacteur de la FR annonçait qu'une société formée en Ecosse, avec société auxiliaire à Londres, avait comme but exclusif d'évangéliser les Canadiens français. Elle recherchait des missionnaires en Suisse.

82 FR (16 août 1840$)$.

ss FR (11 novembre 1838) : 531. Lettre de Mme Feller, 4 mai 1838. 
$\mathrm{Au}$ dire des membres de la Montreal Bible Society, la situation politique défavorisait leur travail ${ }^{84}$; un missionnaire wesleyen de langue anglaise travaillant dans la région d'Odell Town, en 1837-38, confirmait ces difficultés: "But the demoralizing effects of the rebellion militated very much against us, and prevented the World of God of having free course [...] all the means of grace in use among us, were regularly administered; but although they were tolerably well attended, it was easy to perceive that iniquity abounded, and the love of many waxed cold ${ }^{85}$."

Il est aussi certain que l'anticléricalisme nouvellement apparu dans les couches populaires pouvait laisser prévoir un meilleur succès. Mais plus que de cette situation, l'action évangélique des Anglais de Montréal s'inspirait d'un sentiment général de crainte de la reprise des événements révolutionnaires qui avaient failli compromettre leur situation politique et sociale au BasCanada. Profondément choquée par ce qui venait de se produire, la majorité anglaise de Montréal regrettait amèrement que les Canadiens français n'aient pas été assimilés. Désormais, croyaitelle, seule leur conversion au protestantisme pourrait garantir la cohésion et l'unanimité entre les deux groupes ethniques.

C'est dans cette atmosphère que le révérend James Thompson fut nommé en 1838, agent de la British and Foreign Bible Society ${ }^{86}$, auprès de la Société auxiliaire montréalaise. Il remarquait en décembre: "But we see, although somewhat darkly, that even these civil commotions will, in effect, be attendent or followed by good effects, as respects the Kingdom of Christ, and also as respects the Empire to which those colonies belong ${ }^{87}$." Pour lui, comme pour la dizaine d'autres Anglais, pasteurs et laïcs de différentes dénominations religieuses de Montréal qui s'étaient réunis sur son invitation, le 13 février 1839, pour fonder la French Canadian Missionary Society, il était évident que la consolidation du pouvoir britannique et la protestantisation des Canadiens français ne pouvaient être dissociées.

84 Monthly extraets of the correspondance of the British and Foreign Bible Society (février 1839), 678.

85 The autobiography of a Wesleyan Methodist missionary, formerly a Roman Catholic, containing an account of his conversion from romanism, and his reception into the Wesleyan... also a reminiscence of nearly twenty five year's itinerary in the North American provinces (Montréal, 1856). 115 .

86 Organisme "interdénominationnel" fondé en mars 1804 qui a donné naissance à la Montreal Bible Society.

87 Monthly extracts of the correspondance of the British... (février 1839), 678. 
Ils pensaient que les pratiques religieuses des Canadiens français étaient la cause de leurs retards au plan politique, écono mique et social. A titre d'exemple, en 1839, un presbytérien voulant stimuler ses coreligionnaires pour l'évangélisation des Canadiens français formulait ces arguments:

We have during the last eighteen months seen them rise again and again as one man to cast off by force of arms the Government which from a state of vassalage had raised them to the immunities and honours of free men; and ought we to wonder, that, they being destitute of that religion which alone can purify and fortify conscience, should be founded destitute also of those virtues which are essential to the very existence of civil society? ${ }^{88}$.

Vers la même époque, le secrétaire de la French Canadian Missionary Society, James Court, dans une circulaire destinée à expliquer aux Anglais les objectifs et le fonctionnement de cette nouvelle société missionnaire, tirait cette conclusion sur l'état du groupe à convertir: "So long as the French Canadians remain in the present thoroughly ignorant and degraded state, it will be impracticable to carry on successfully a government among them founded on the principles of the British constitution [...] It may be reasonably anticipated that under the Divine Blessing righteousness, peace, and good government, will here long be established." ${ }^{89}$ Dans une adresse aux Eglises évangéliques de GrandeBretagne. les membres de la FCMS ${ }^{90}$, désirant obtenir leur assistance, prirent soin d'abord de leur rappeler les désordres révolutionnaires qu'ils avaient peut-être déjà oubliés et les dépenses considérables que le rétablissement de l'ordre leur avait occasionnées, pour exposer ensuite une des raisons de la nécessité de collaborer à leur évangélisation: "[ . . . there can be little difficulty in arriving at the conclusion that many of the evils by which we have been scourged have had their origin in the ignorance, superstition, and wickedness incident to our deprave nature unalliviated by the possession of the Gospel and that these characteristics are but augmented and stereotyped by the influence of Romanism". ${ }^{91}$ Citons enfin cet autre exemple parmi

\footnotetext{
88 The Canadian Christian Examiner and Presbyterian Review, III (1839) : 164.

89 Archives de l'Eglise Unie du Canada (AEUC), Circulaire du 12 avril 1839, reliée au ler volume du Rapport annuel de la FCMS.

90 French Canadian Missionary Society.

${ }^{91}$ AEUC, The Minute Book of FCMS, vol. I (9 novembre 1844).
} 
tant d'autres ${ }^{92}$ : en 1848 , lors de l'assemblée annuelle, un membre éminent du comité de direction fait voter cette résolution: "That as the late famine in Ireland, and the distress and crime that are now so prevalent there are chiefly to be ascribed to the influence of the Popery in that country, and to be regarded as it legitimate fruits, all who deprecate the existence of such scenes in this Province [Canada Est] ought to aid the efforts of this Society." 93

Les fondateurs de la nouvelle société missionnaire de Montréal ne craignaient pas d'afficher publiquement devant leurs compatriotes du Canada et de Grande-Bretagne l'égoïsme des motifs de leur prosélytisme, plus soucieux d'assurer leur propre sécurité politique et sociale que le salut des Canadiens français, car ils avaient conscience de s'adresser à des gens qui ne doutaient pas de leur croyance à la supériorité des vertus civiles que leur conférait la pratique du protestantisme.

Toutefois, si un grand nombre d'Anglais du Bas-Canada cultivaient ce mythe et considéraient la pratique religieuse des Canadiens français et l'influence du clergé comme la principale explication de leur révolte et de leur retard au plan économique et social, les Anglicans et ceux qui détenaient des postes administratifs ne partageaient pas ce point de vue. Ceux-là avaient d'abord cru, surtout au lendemain de la révolution américaine, que seul l'anglicanisme pouvait assurer la fidélité à la couronne britannique. Mais depuis une trentaine d'années, les événements - plus particulièrement les nombreuses preuves de loyalisme du clergé catholique et l'impossibilité d'établir la religion anglicane - les avaient forcés à reviser leur opinion. A l'instar de Durham et des autres gouverneurs ${ }^{94}$, ils pensaient maintenant, en dépit de la rébellion, que l'influence du clergé catholique était indispensable au maintien de l'ordre et qu'il ne fallait pas chercher

92 J. I. Cooper, "The early editorial policy of the Montreal Witness", in RSHC (1947): 55, indique de nombreuses références à ce journal rédigé par John Dougall, membre du comité de direction de la FCMS, où l'auteur entremêle les raisons religieuses et politiques pour persuader ses compatriotes de se consacrer à la conversion des Canadiens français.

93 The Missionary Record (février 1848). Proposée par le rév. W. Taylor et secondée par le rév. J. Girwood.

94 Soulignons que certains gouverneurs forcés par les circonstances politiques durent s'astreindre à ne pas dévoiler, ni mettre en pratique leurs véritables sentiments vis-à-vis du catholicisme; ils ne pensaient pas moins, tel que l'écrit le sulpicien Jean-Baptiste Thavenet à propos de lord Bathurst, que "[...] la religion catholique est un obstacle au développement de l'intelligence, de l'industrie et du commerce et que la religion protestante peut faire seule le bonheur des peuples [...]". (Thavenet à l'évêque de Québec, Paris, 15 mars 1827, in RAPQ (1933-34) : 340-41). 
à l'affaiblir en travaillant à la conversion des catholiques. Du reste, au dire d'un membre de la FCMS, ceux qui s'objectaient à leur prosélytisme considéraient le catholicisme comme "an amiable and imposing system, well adapted for the poorer class, and as capable as their own, if sincerely followed to bring their professors to heaven" ${ }^{95}$. Ce point de vue était défendu par les Anglais les plus influents, de telle sorte que la FC.MS ne reçut jamais officiellement ni l'appui ni l'encouragement du gouvernement britannique.

Le prosélytisme des missionnaires de la FCMS fut d'abord orienté vers les régions où la rébellion avait eu quelqu'emprise. Louis Roussy, de la mission de Grande-Ligne, invité à collaborer avec eux pour choisir le site de leur première mission, confirma en ces termes le bien-fondé du choix de la région de Belle-Rivière, à proximité de St-Eustache: “On sait que c'est dans ce dernier endroit que fut livrée, en 1838, la bataille qui étouffa la révolte des Canadiens dans cette contrée; et l'on peut espérer que les grands maux qu'ils eurent à souffrir dans cette occasion, ont préparé leurs cours à l'Evangile ${ }^{98}$."

Cette conjoncture favorable tirait à sa fin; dorénavant, on ne retrouvera plus dans leurs écrits de semblables remarques, car non seulement le climat politique sous l'Union invitait l'élite à oublier les débats de la période précédente et à laisser au clergé catholique l'influence qu'il réclamait mais aussi ce dernier venait de mettre en branle ce que l'historien Léon Pouliot a appelé "la réaction catholique". Un contemporain, ancien patriote, le notait: "[...] nos prêtres prennent les moyens de fanatiser notre peuple à l'extrême. Depuis que les Pères Oblats (quatre Français importés par l'évêque de Montréal) sont en Canada, ils se transportent d'une paroisse à l'autre et ce sont des retraites ou missions qui s'y font, de trois semaines de longueur." ${ }^{97}$ C'est aussi en faisant allusion à ces retraites, et dans l'ensemble aux diverses manifestations du "réveil religieux", qu'un dirigeant de la FCMS écrivait, en 1842: "Romanism, it would seem, is not in such a state of decrepitude as we have been led to suppose ${ }^{28}$." la FCMS.

95 Occasional Paper, no 7 (19 février 1842). Publié par le comité de 06 FR (6 septembre 1840).

97 RAPQ (1926-27): 247. Lettre de Louis Perrault à Duvernay, 4 février 1842 .

98 Occasional Paper, no 7 (19 février 1842). 
Les évangélistes protestants devaient donc compter sur leurs seules forces, dans un pays hostile. Ils étaient par ailleurs désavantagés par leurs divisions. Mme Feller et Louis Roussy avaient refusé de se joindre à la société montréalaise, de telle sorte qu'ils s'étaient entendus pour se partager le territoire: la FCMS au nord du St-Laurent et l'autre au sud. Mais cette entente subit très tôt des accrocs qui refroidirent passablement leurs relations ${ }^{99}$, et surtout projeta l'image de deux sociétés rivales. En 1847, les anglicans qui, depuis le début du XIXe s'étaient abstenus de faire du prosélytisme auprès des Canadiens français, engageaient le Suisse Daniel Gavin pour desservir l'église française de Sabrevois (près d'Iberville) ${ }^{100}$. A ces trois groupes distincts ${ }^{101}$ qui sollicitaient les catholiques parfois dans un même territoire s'ajoutèrent les méthodistes, à partir de $1855^{102}$, et les presbytériens en $1861^{103}$.

En dépit de ces difficultés et de cette concurrence, le mouvement de protestantisation qui avait pris son essor avec la rébellion fit des progrès constants, du moins jusque vers 1860. La FCMS comptait alors 117 membres de l'Eglise, employait 33 évangélistes, y compris les ministres, les colporteurs et les enseignants qui célébraient le Sabbat dans 12 localités et visitaient la plupart des régions de la province où plus de 600 personnes, adultes et enfants, assistaient à leurs prédications ${ }^{104}$. Vers la même époque, les 20 évangélistes de la mission de Grande-Ligne visitaient 40 localités comprenant 700 membres de l'Eglise et environ " 3,000 convertis" ${ }^{105}$. Et ces chiffres, à leurs dires, n'indiquaient pas les

${ }^{99}$ AEUC, The Minute Book of the FCMS, vol. I, 1837-47, 10 aout 1841,14 mai 1844, 11 juin 1844, 26 février 1847. Voir aussi Duclos, op. cit., 195-197 et le 8e Rapport annuel de la FCMS (janvier 1847).

100 Gavin avait été ordonné en 1847. L'année suivante la veuve de Plenderleath Christie fit construire l'église de Sabrevois sur ses terres.

101 La Mission de Grande-Ligne s'était affiliée à la Canada Baptist Missionary Society de Montréal en 1845 (Missionary Record, août 1845). 102 Paul Villard, Up to the Light. The Story of the French protestantism (c 1928), 78-79.

103 Le ministre J. E. Tanner quitta la FCMS avec 26 membres de sa communauté pour s'affilier à l'Eglise presbytérienne.

10421 e Rapport annuel de la FCMS (1860). A ces évangélistes, il faut ajouter leurs épouses qui de temps en temps rendent des services au niveau du colportage et de l'enseignement. La même chose se produit à la Mission de Grande-Ligne.

105 Théodore Lafleur, A semi-centennial historical sketch of the Grande

Ligne Mission (...) (Montréal, 1885), 32. Ce chiffre de "3,000 convertis" - traduction de l'anglais "converts" - me semble excessif; il réfère peut-être au nombre de personnes, adultes et enfants, qui dans chacune des localités ont assisté à leurs réunions de prières ou ont reçu avec sympathie leur littérature de colportage. Ces gens ne sont pas tous des convertis. Du reste, la FCMS est volontairement imprécise quant aux résultats de son 
résultats réels de leur travail ${ }^{106}$, car l'émigration aux Etats-Unis qui avait commencé à décimer la population du Québec, attirait davantage ces convertis recrutés en majorité dans les classes laborieuses, et par surcroît bannis de la société canadiennefrançaise.

travail, prétextant que des Anglais peu sympathiques à la cause mettent en relations le nombre de convertis et les sommes d'argent investies. Pour cette raison et pour d'autres évidentes, il est probable que la Mission de Grande-Ligne ait eu le même comportement.

106 Les évêques de Saint-Hyacinthe, Québec, Montréal et Trois-Rivières confirment, dans leurs rapports à la Propagande, le dynamisme et les succès relatifs des sectes protestantes. Si l'évêque de Saint-Hyacinthe minimise leurs gains depuis le début à "quelques centaines d'Apostats" (Arch. de la Prop., S.R.C. Am. Sett., 1862-65, Rapport sur le Diocèse de Saint-Hyacinthe [...], 11 nov. 1864), celui de Montréal en donne une description qui me semble plus révélatrice de leurs influences réelles: "En plusieurs endroits, écrit-il, des Prédicateurs Evangélistes [...] répandent des Bibles falsifiées et des tracts empoisonnés [...]; ils construisent des écoles hérétiques; ils distribuent à pleines mains de l'argent pour gagner les pauvres; dans des hospices, ils nourrissent et donnent l'éducation à des enfants, garçons ou filles, orphelins ou abandonnés; ils reçoivent ou instruisent gratuitement les jeunes dans leurs collèges; ils construisent des chapelles et autres salles publiques; ils achètent des domaines où ils établissent des cultivateurs et des pauvres, et ils se les attachent sous le prétexte honnête de leur donner de quoi vivre; ils visitent les maisons, les chemins publics, les prisons, les chars et les navires, pour y répandre leurs livres, discours et sermons. Dans les endroits où il $\mathrm{y}$ a des divisions parmi les catholiques, ces ministres font des visites et ils déclament contre les richesses de l'Eglise, le pouvoir temporel du Souverain Pontife. l'autorité spirituelle des Pasteurs. - Les fidèles sont avertis souvent de les éviter; on multiplie les Collèges et les Ecoles; on édifie en divers lieux des Orphelinats, des hospices pour les vieillards infirmes et les veuves délaissées. Dans les villes et les campagnes, on organise des couvents pour les filles pauvres; on fait des exercices spirituels (retraites) pour les fidèles [...]" (Traduction de l'original en latin, Arch. de la Prop., S.R.C., Am. Sett., 1862-65, Secunda Relatio episcope Marianopolitanensis (...), Rome, 27 avril 1862). 